EPJ Web of Conferences 33, 04001 (2012)

DOI: $10.1051 /$ epjconf/20123304001

(C) Owned by the authors, published by EDP Sciences, 2012

\title{
Fundamental properties of and transition to a fully renewable pan-European power system
}

\author{
G.B. Andresen ${ }^{1, \mathrm{a}}$, M.G. Rasmussen ${ }^{1,2}$, R.A. Rodriguez ${ }^{1}$, S. Becker ${ }^{1,3}$ and M. Greiner $^{1}$ \\ ${ }^{1}$ Department of Engineering, Aarhus University, DK-8000 Aarhus C, Denmark. \\ ${ }^{2}$ Lehrstuhl für Mathematische Statistik, Technische Universität München, \\ DE-85748 Garching bei München, Germany. \\ ${ }^{3}$ FIAS Frankfurt, University of Frankfurt, DE-60438 Frankfurt am Main, Germany.
}

\begin{abstract}
We introduce a top-down stylized model to analyse the impact of a transition to a European power system based only on wind and solar power. Wind and solar power generation is calculated from high-resolution weather data and based on the country specific electricity demand alone, we introduce a model of the conventional power system that facilitates simple spatio-temporal modelling of its macroscopic behaviour without direct reference to the underlying technological, economical, and political development in the system. Using this model, we find that wind and solar power generation can replace conventional power generation and power capacity to a large degree if power transmission across the continent is made possible.
\end{abstract}

\section{Introduction}

The design of a fully renewable power system for future Europe depends on the weather. The weather determines how much wind and solar power generation is best for Europe, how much and what kinds of storage [1,3-4], balancing [2-4] and power transmission [5-7] are needed, and how much fossil and nuclear power generation is still required during the transitional phase. Simple spatio-temporal modelling based on high-resolution weather data, electricity consumption time series, and the physics of complex networks provide quantitative answers to these important questions. Included in the analysis are 27 European countries, and we model hourly conventional and renewable power generation over a continuous period of 8 years.

In order to find the optimal transitional path, we introduce a top-down stylized model of the present-day European power system. The model facilitates simple spatio-temporal modelling of the macroscopic behaviour of the power system without direct reference to the underlying technological, economical, and political development in the system. A set of simple rules, aimed at minimizing the conventional power system, are then used to calculate the power system composition and dynamics for a fixed amount and mix of renewable power production [6-7]. In addition, the model calculations provide direct and quantitative answers to the consequences and the large potential benefits of introducing a strong continental power grid in the European system.

\footnotetext{
a e-mail : gba@ase.au.dk
}

This is an Open Access article distributed under the terms of the Creative Commons Attribution License 2.0, which permits unrestricted use, distribution, and reproduction in any medium, provided the original work is properly cited. 


\section{Mixing wind and sun}

\subsection{Data}
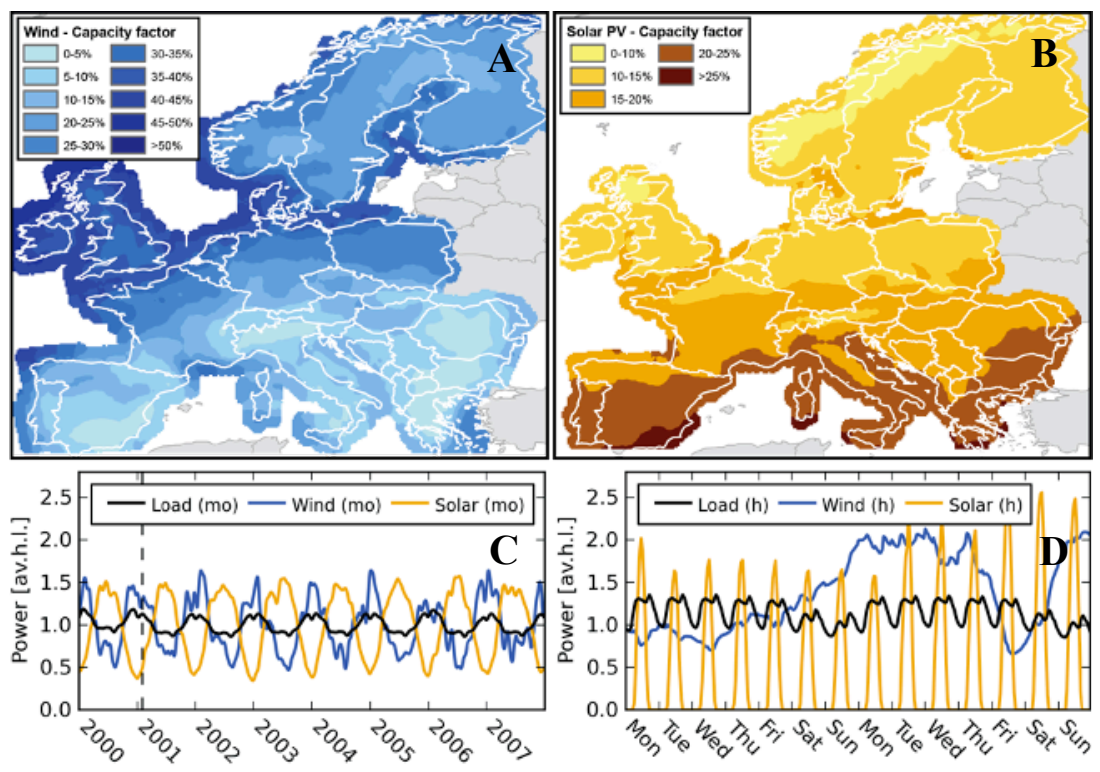

Fig. 1. Wind and solar power generation is derived from historical weather Panels A and B illustrate the geographical extend of the data. In colour we show: (A) average wind power output scaled to installed peak capacity (wind power capacity factor), and (B) average solar PV power output scaled to installed peak capacity (solar PV power capacity factor). Panels C and D show normalized monthly and hourly averages of wind and solar PV power generation and electricity demand aggregated for all 27 countries. [6-7]

Normalized wind and solar power generation is derived directly from historical weather data with a spatial resolution of $50 \times 50 \mathrm{~km} 2$ and a temporal resolution of 1 hour. The data set covers both on and offshore regions for 27 European countries and spans the 8-year period 2000-2007. The geographic extend of the data set is shown in Fig. 1, where the calculated potentials for wind and solar power are indicated by their respective average capacity factors. Also shown in the figure are examples of aggregated monthly and hourly wind, solar PV and load time series for Europe.

To obtain the absolute power output at a given location we apply capacity scaling factors to each grid cell. The scaling factors reflect the assumed installed wind and solar power capacity at that location, and combined; all such scaling factors form maps of the installed wind and solar power capacities. These layouts are based on the national EU-2020 targets of Europe where emphasis is given to the most attractive European sites.

The electricity demand (or load) for all 27 European countries is obtained on a regional basis with hourly resolution. Regions typically consist of the domain of a single transmission system operator (TSO) and inside each region power is assumed to flow freely. In this analysis, we generalize further, and assume that power can flow freely within the borders of each country or within Europe.

Additional details on the origin and processing of the weather and load data can be found in the appendix of [1].

\subsection{A seasonal optimal mix of wind and solar PV}

The modelling approach, described here, was first applied to a fully renewable European power system, where wind and solar PV is assumed to generate exactly enough power to cover the load on 


\section{$2^{\text {nd }}$ European Energy Conference}

average. In order to make generation match the demand in such a system, storage is introduced too. The objective of the analysis is then to determine the minimum sufficient energy storage capacity that allows excess to be shifted in time to cover all hours with shortage of wind and solar PV power generation. The results of this analysis are described in [1], and in [2] they are extended to also include a system with an over installation of wind and solar PV.

Figure 2, shows some of the key results of the analysis. In the left panel, we see that an optimal mix of wind and solar power can reduce the required storage capacity from about 3 to 1.5 average load month for a system, where the average wind and solar power generation exactly matches the average load. Here, one average load month for Europe corresponds to one twelfth of the annual consumption of about $3240 \mathrm{TWh}$ in 2007. Depending on the efficiency of the storage technology used, a total storage energy capacity of between 330 and $400 \mathrm{TWh}$ is required to ensure security of supply in this case. Such a large storage energy capacity can most likely only be realized through a strong coupling to the gas infrastructure. However, as shown in the left panel of Figure 2 an over installation of wind and solar PV can significantly reduce the required storage energy capacity. At an over installation of $50 \%$, the required capacity is reduced by about an order of magnitude to between 30 and $50 \mathrm{TWh}$.
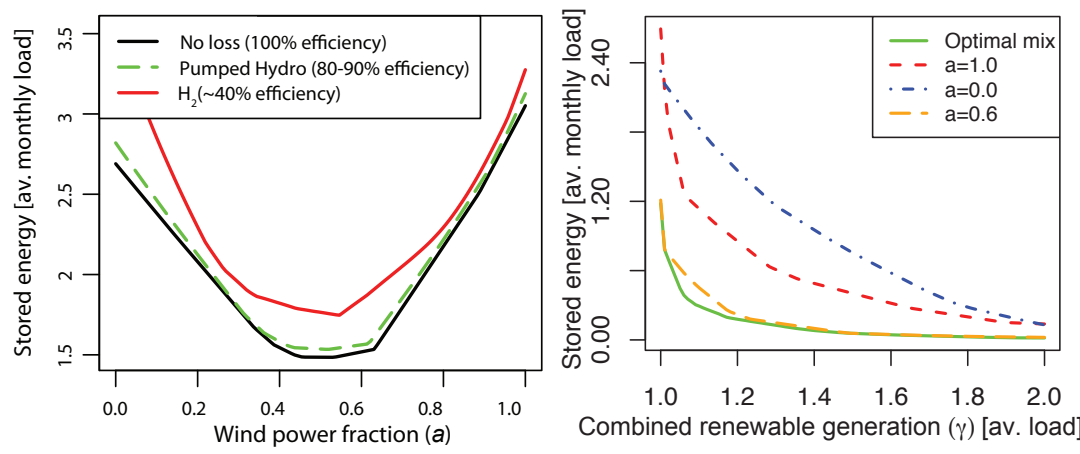

Fig. 2. Left panel: Comparison of the maximum stored energies for an idealized storage (black), pumped hydro (green) and hydrogen storage (red) as a function of the wind fraction (a). The unit of the stored energy is in average monthly load. Right panel: Storage energy capacity as a function of the excess generation $(\gamma)$ and for different shares a of wind power generation (a). The storage efficiencies have been set to 1. [1-2]

In [3-4], we extend the model to also include the case where the average wind and solar power generation is smaller than the average load. Some of the results of this analysis are shown in Figure 3 . The left panel of the figure shows how the required storage energy capacity grows rapidly at a penetration of about $50 \%$ and peaks at a penetration of $100 \%$. The right panel of the figure shows the effect of having a less than sufficient storage energy capacity for 75\%,100\% and $125 \%$ penetration levels. From the figure it is clear that the gain that can be achieved with even a relatively small energy storage capacity can be significant. In fact, a storage energy capacity of about 6 average load hours provides approximately half the benefit of the much larger sufficient capacity, which was shown to be at least 1.5 average load months above. 

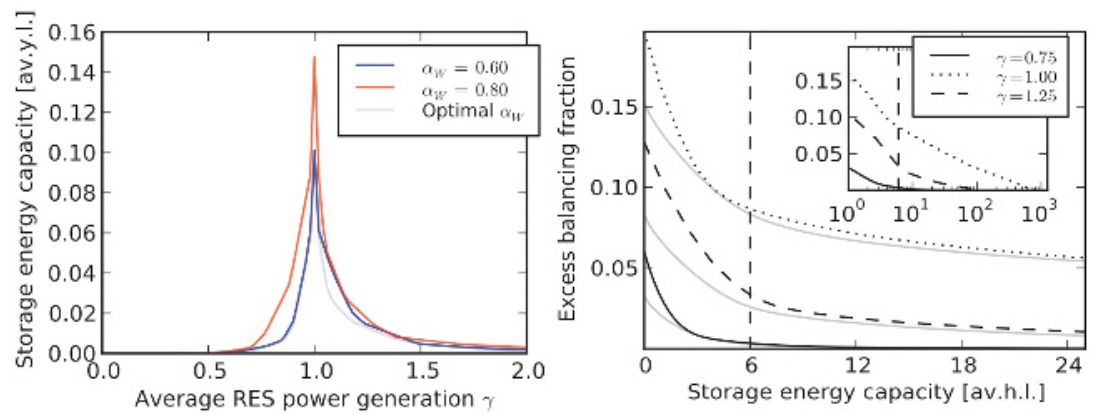

Fig. 3. Left panel: Minimum sufficient storage energy capacity vs. average RES power generation $(\gamma)$ for different fractions of wind power $\left(\alpha_{w}\right)$. Right panel: Excess balancing vs. a constrained storage energy capacity. [3-4].

\section{Balancing the residual load}

In a power system with a high share of weather driven renewable power generation, the supporting flexible system must satisfy the residual load that remains after subtracting the available power generation from the actual load. Generally, the residual load must be satisfied for each country, but depending on the capacity of cross-border transmission lines, flexible resources and renewable power generation can be shared between some or all countries in order to do so.

In the following, we define the supporting system and propose a set of simple operational rules which allows power generation to be assigned with different levels of flexibility similar to those associated with base load, intermediate load, and peak load power generation. Doing so allows us to make a bridge between the traditional power system of today and a future power system with very high shares of weather driven power generation and provides a tool to estimate at what level weather driven power generation is able to replace flexible conventional power generation, in particular, base load power generation.

In order to identify a natural distinction between different levels of flexibility in the traditional power system, we identify a set of three simple patterns of repetition found in the electricity load time series for all 27 countries in the data set. These are: a seasonal, a weekly, and a daily pattern. The seasonal pattern generally distinguish winter from summer, but varies significantly from country to country, as it depends on seasonal changes in the local environment and events such as national holidays. The weekly pattern distinguishes weekend days from workdays with a lower average load during the former, for all countries. Finally, the daily pattern is characterized by large night-day variations in the load, and distinct peak-hours, typically, during morning and evening. As a rule of thumb, the difference between the maximum and the minimum load hour during any 24-hour window amounts to about $20-40 \%$ of the daily maximum, depending on the country. As an example, Figure 1 illustrates the seasonal, weekly, and daily patterns of the combined European load. Associated with each of the three patterns is a distinct time scale and amplitude. In order to meet the electricity demand, the underlying power system must have sufficient regulation power that can be dispatched at the appropriate time scales.

Combining the properties of the three patterns identified in the load time series with the fact that different power plants have different ability to regulate their output, we define a simple model of the present-day power system: First, regulating capacity which can be dispatched on time scales in the interval 1 to 24 hours is associated with the daily pattern, and a country specific minimum sufficient peak capacity of an intra-day flexible system is derived directly from the high-frequency component of the load time series. The remaining low frequency component of the load time series is then analysed in a similar way to find the minimum sufficient peak capacity that can be dispatched on time scales in the interval 1 to 7 days. This capacity is associated with an intra-week subsystem. Finally, a minimum sufficient peak capacity that can be dispatched on time scales longer than one 
week, remains. This capacity is associated with a seasonal power subsystem.

The simplified power system, described above, makes use of the smallest possible set of power capacities in the sense that the minimum capacity for each subsystem is identified in order of the most to the least flexible system. The capacities are designed to fully cover the load during $99 \%$ of the 70128-hour-long data set and to avoid any excess power generation when applied to the load curve they are derived from. The rules allow any residual load with a maximum smaller than or equal to the combined power of the three flexible systems to be met, and ensure that the most flexible systems are utilized to minimize excess production.

\subsection{Results}

The power capacities of the three present-day conventional subsystems are calculated from the electricity demand curve for each individual country as, presently, only few European countries rely on transmission as a means of balancing the electricity demand. Summed over all countries this leads to aggregated capacities of: $272 \mathrm{GW}$ for the seasonal system, $133 \mathrm{GW}$ for the intra-week system, and $165 \mathrm{GW}$ for the intra-day system.

In the following we distinguish between two cases: In the first case, we assume the European power system to be the sum of isolated national power systems. We name this case closed borders. In the second case, dubbed open borders, we assume free flow of power between all countries.

Figure 4 shows the average power generation and power capacities of the seasonal, intra-week, and intra-day power systems as a function of the total wind and solar power penetration for closed (left panel) and open borders (right panel). The average wind and solar power generation is shown as well. The corresponding minimum power capacities of the flexible systems are shown in the inset of the figure. Both in the case of open and closed borders, about $25 \%$ renewable power can be included in the power system without incurring excess generation. However, in the case of closed borders this only leads to a small decrease in the required power capacity of the seasonal subsystem. In contrast, about $30 \%$ of the installed seasonal capacity can be shut-down in the case of open borders, most of which can be attributed directly to installed capacities of wind and solar power, while a smaller amount is a consequence of sharing balancing capacities and reducing fluctuations in demand and generation through aggregation. When the average wind and solar power generation is between $25 \%$ to $75 \%$ of the average demand, the dominant source of fluctuations in the residual load changes from fluctuations in electricity demand to fluctuations caused by wind and solar power generation. In this interval, the seasonal capacity decrease at a lower rate for both open and closed borders, and complete integration of all renewable power generation can only be achieved in the case of open borders, high-lighting the need for cross-border power transmission. Beyond $75 \%$, excess power generation is also present in the case of open borders, and when the average renewable generation exceeds $100 \%$ of the demand, excess power generation cannot be avoided as annual generation exceeds the demand. The rate of decrease of seasonal power capacity stabilizes at approximately $75 \%$ for both open and closed borders. However, in the case of closed borders the rate of decrease has an asymptotic behaviour and never falls below a certain point (not shown), whereas for the open borders the seasonal capacity becomes zero at a combined wind and solar penetration of approximately $125 \%$. For a penetration of approximately $160 \%$, the capacity of the intra-week system too becomes zero, leaving the intra-day system as the sole flexible supporting system for wind and solar power generation. 

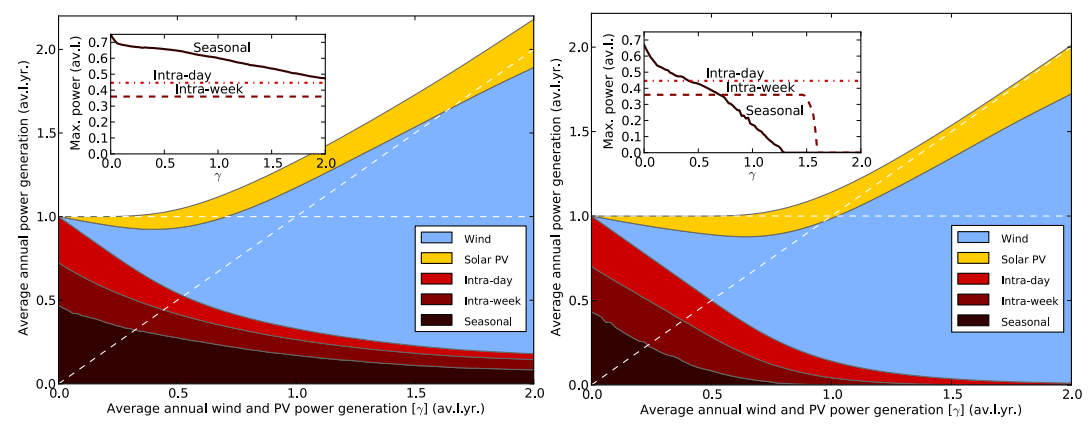

Fig. 4. Average annual power generation of the flexible intra-day, intra-week, and seasonal systems vs. average annual combined wind and solar PV power generation in units of an average load year (av.l.yr.). The average power generation of wind and solar are shown as well. The inset shows the corresponding power capacities of the three flexible systems. Two cases are shown: closed borders (left panel), i.e., no power transmission across the national borders, and, open borders (right panel), i.e., unlimited power transmission between all countries. $[6,7]$

\section{Transmission of renewables}

Here, we consider a fully renewable scenario, where each of the 27 European countries are given a $100 \%$ renewable penetration and a wind-solar mix with $73 \%$ wind and $27 \%$ solar power generation. This mix is found to minimize the mean residual load for the countries on average, but as the variation around the mean is small, a single value for all countries is chosen for simplicity.

If no transmission between the countries is considered, these choices results in a mean residual load of $25 \%$ of the mean load for the countries on average. This means that, although each country has a $100 \%$ renewables penetration, a quarter of all generation has to be discarded due to excess production. If on the other hand, unlimited transmission capacities are assumed, the mean residual load is reduced to $15 \%$. The difference is the maximum benefit that can be obtained from a panEuropean transmission network in the fully renewable scenario considered here.

To quantify the transmission capacities required to achieve the maximum benefit, described above, and to estimate the benefit that can be realised with a less than ideal transmission system, a constrained power flow analysis is required. For this purpose we have developed a numerical tool to solve the security constrained DC power flow in the network [5]. Using this tool, the power flows in the network are calculated to minimize total dissipation for each hour in the 8-year-long data series.

The results are shown in Figure 5 for two different ways of scaling the transmission capacity layout in the network. The upper curve (blue) shows the effect of ramping up today's capacities in a linear fashion. The lower curve (green) represents a more optimal capacity layout based on the layout required to achieve a copper plate like flow. In both cases, the curves are highly convex, indicating that a large fraction of the maximum benefit can be achieved with a less than ideal transmission capacity layout. More specifically, current installed capacities could already provide roughly a third of the maximum benefit, and doubling the total installed capacity over Europe could further reduce the balancing required by a similar amount. A 5 fold increase in total transmission capacity would allow unimpeded power flow during $99 \%$ of all hours, but to achieve the full benefit of transmission, about 10 times the total capacity installed today would have to be deployed. 


\section{$2^{\text {nd }}$ European Energy Conference}
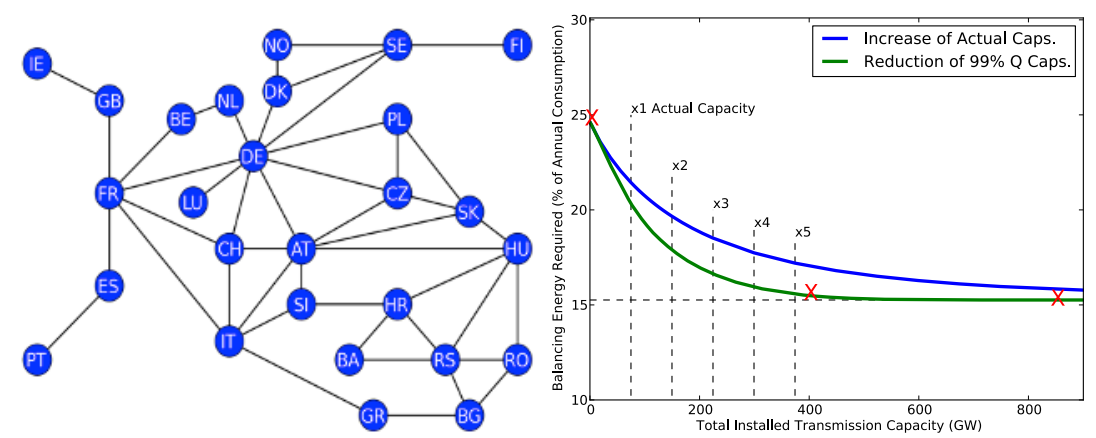

Fig. 5. Left panel: The transmission lines connecting the 27 European countries. This network topology resembles that of the present day European power system. Right panel: As a result of the DC power flow calculation, the average balancing energy of the nodes is shown as a function of the total installed transmission capacity in the network. The significance of the two different curves joining the extreme points is discussed in the text. [5]

\section{References}

1. D. Heide et al., Renewable Energy 35, 2483 (2010).

2. D. Heide et al., Renewable Energy 36, 2515 (2011).

3. M.G. Rasmussen, G.B. Andresen and M. Greiner, "Storage and balancing synergies in a fully renewable pan-European power system" ", Manuscript in review.

4. M.G. Rasmussen, G.B. Andresen, D. Heide and M. Greiner, Proceedings of the 10th International Workshop on Large-Scale Integration of Wind Power into Power Systems as well as on Transmission Networks for Offshore Wind Power Plants, Energynautics, 682-684 (2011).

5. R.A. Rodriguez, U. Poulsen, G.B. Andresen, D. Heide and M. Greiner, "Transmission within a fully renewable pan-European power system", Manuscript in preparation.

6. G.B. Andresen, M.G. Rasmussen and M. Greiner, "Transition to a fully renewable power system for Europe", Manuscript in preparation.

7. G.B. Andresen, M.G. Rasmussen, D. Heide and M. Greiner, Proceedings of the 10th International Workshop on Large-Scale Integration of Wind Power into Power Systems as well as on Transmission Networks for Offshore Wind Power Plants, Energynautics, 322-325 (2011). 